\title{
Opening the Black Box of Innovation Processes in Virtual Communities
}

\author{
Meera Sarma \\ Northumbria University, UK
}

\section{Abstract}

Communities involved in free and open source activities possess special structural and processual characteristics that are conducive to innovative product development on-line. We suggest that this form of innovative, knowledgegenerating, community may benefit from being seen within the framework of virtual process research using Virtual Ethnography (VE). In order to analyse the virtual ethnographic observations, innovative software research tools, such as the "IRC Conversation Map', had to be devised. The investigation explored the structural and temporal dynamics of the community through the examination of interactions between members, as they unfolded in virtual space. In this way, we show how the themes that constitute the innovation process emerge from everyday routines that members of the hacker community perform. This paper contributes to the methodology of process research by exploring strategies that allow qualitative researchers to examine virtual organized activities through a process lens.

Keywords: Hackers, Innovation, Open Source Software, Innovation JEL classification: O3

\section{Introduction}

The growth of the internet has led to the formation of new forms of social exchange, creating what are generically known as 'virtual communities' (Klang \& Olsson, 1999 Numerous articles have emerged on virtual firms, organizations, and work teams. However, there is little theoretical insight into the different ways that virtual communities can work. The Open Source Software (OSS) community provides one of the most intriguing examples of how virtual communities can be innovative on-line.

The diversity of communities, particularly in terms of their organization, control and development, makes generalization difficult particularly in understanding how sequences of events unfold in the virtual environment. This paper reveals the complexities of understanding process research and examines how the innovation process in the virtual context, although fluid and spatially and temporally dispersed can be perceived and analyzed, allowing us to explore theoretical qualities of the phenomenon.

Open Source Software (OSS) is considered to be one of the most promising new alternatives to monopolistic proprietary software. The significance of OSS is reflected in both the degree of openness that allows anybody to participate and the innovative capabilities that arise from an infrastructure that is independent of and different from proprietary forms. Existing explanations of how OSS has taken the creative lead in the development of technologies have gone some way to clarifying the existence of the phenomenon, but they fail to address a number of significant questions about the process of innovation; these questions call for in-depth qualitative research (Von Krogh et al., 2003). 
Despite a growing number of studies investigating OSS from various perspectives, questions concerning the process of innovation have been addressed in far less detail. Further, little is known about how people in these communities develop innovative software across dispersed geographical settings, or about what software processes, structural characteristics and knowledge sharing practices are necessary for their success. In researching these sorts of issues, we aim to contribute to the literature on OSS communities. We argue that understanding how innovation occurs and how the pace of innovation is maintained in the virtual environment calls for a theoretical framework that develops new concepts and explanatory relationships, and builds on existing theories of OSS development.

We seek to answer the following question: How does the process of innovation unfold in the virtual environment?

From an innovation perspective, what needs to be uncovered is why and how individuals contribute to the innovation process (Von Krogh, et al., 2003). Scholars have identified reputation (Raymond, 1999), the underlying free software ideology and creativity (Stallman, 2001), as factors influencing participation. Research into these factors provides valuable evidence about why individuals participate but does not explain how the activities of members and the knowledge processes they engender influence software development.

\section{Methodology}

Through a series of face-to-face interviews, it was initially possible to identify and analyze the broad themes and patterns of interaction within the OSS community. However, Virtual Ethnographic (VE) methods allow researchers to make a detailed empirical examination of the innovation process as it happens through virtual chatroom exchanges.

We picked a three-month period in the community's product release cycle. By choosing a random time period we wanted to observe and provide details of practices of problem solving in virtual space, procedures of bug reporting, occurrences of chaos and conflict, so as to highlight the procedural, contextual and structural backdrop to the innovation process. We examined not just the events unfolding but also the interaction between members in day-to-day activities. The OSS community undergoes cycles of product release, which provide a rhythm for the particular social system. There are numerous social activities taking place before and after a release, and we have tried to capture the social activities that unfolded during the period of observation. We investigated the chat room logs of nine OSS projects.

\section{Results}

The chatroom conversation map (developed in this paper) offers a dynamic integrating lens to trace the activities of individuals, groups, and community over a period of time. It allowed for a dynamic micro/macro perspective on the activities over any period of time, tracking the trajectory of participants in a discussion. It enables us to open the black box of the structural shifts in the community that appear to the outside observer as a single stream of activities. We are able explore the social networks of the OSS community by drilling down into the micro processes of interactions between the members, using different criteria to analyze the flow and content of the interactions. It provides a means of visualizing the communication networks of the OSS community and their evolution over time.

The following forms of analysis are used for the study: 
1. Keyword search analysis this not only takes into consideration the situated context in which the software development occurs, but also reveals what occurs in a given spatial and temporal dimension. Such analysis is carried out using 'keyword searches' to trace particular aspects of communication that offers critical insights into the context in which the software is being produced. The keyword searches are performed with the development of the IRC Conversation Map, described in the following section.

2. Structural Analysis: the structural data explicate how the process of innovation unfolds over time and helps to investigate the mesh of interactions that takes place. Through Social Network Analysis (SNA), it is possible to get inside the black box of the innovation process and validate the data collected from the interviews.

Once the logs were collected, the IRC Conversation map was set up and the analysis was conducted. First, for structural analysis of the community, particular members were selected, who occupied crucial positions in the network and were active participants. Their interactions with other members in the social network were probed. Further, members holding independent discussions in the different social network graphs, forming independent networks, were also analysed. This corresponds to the chronological and processual aspects of the analysis, as discussed earlier.

Second, to understand how knowledge was shared and disseminated in the community and to understand particular aspects that emerged from the interviews, such as flame wars and forking, we used keyword analysis. Keyword searches were conducted for the entire network of the two chosen projects. Not only does this capture novel information that structural analysis overlooks, but specific elements of interactions are identified. The keyword analysis allows us to understand better the contextual and the processual elements of the study. Following is the list of keywords for which a search was conducted, for both the epiphany and gtk+ projects.

Keyword search term: meaning inferred

a. learn/question/experiment: self learning

b. hack/hacker: identity

c. Problem /Solution: Joint problem solving

d. Idea: Idea generation

e. Review: Code review and development

f. Feedback: innovation adoption and final release

g. Freeze: TBR (time based release)

h. Stable/unstable release: TBR

i. Merge /Branch/ Fork: Parallel innovation

j. Flame: Conflict

The keyword search analysis identifies particular words or phrases in the log file. It is also extremely useful in identifying the context in which a particular word is used, thus clarifying the meaning of a particular action in conjunction with the context in which it is produced.

Through analysis of processual data, we can show how virtual activities not only reflect the constraints of the community structure but also shape it and in doing so give form to the process of innovation as it unfolds.

The following figures illustrate the data extracted and the use of the IRC map. 
Figure 1

An Example of a Social Network

A Social Network Diagram for an IRC Channel

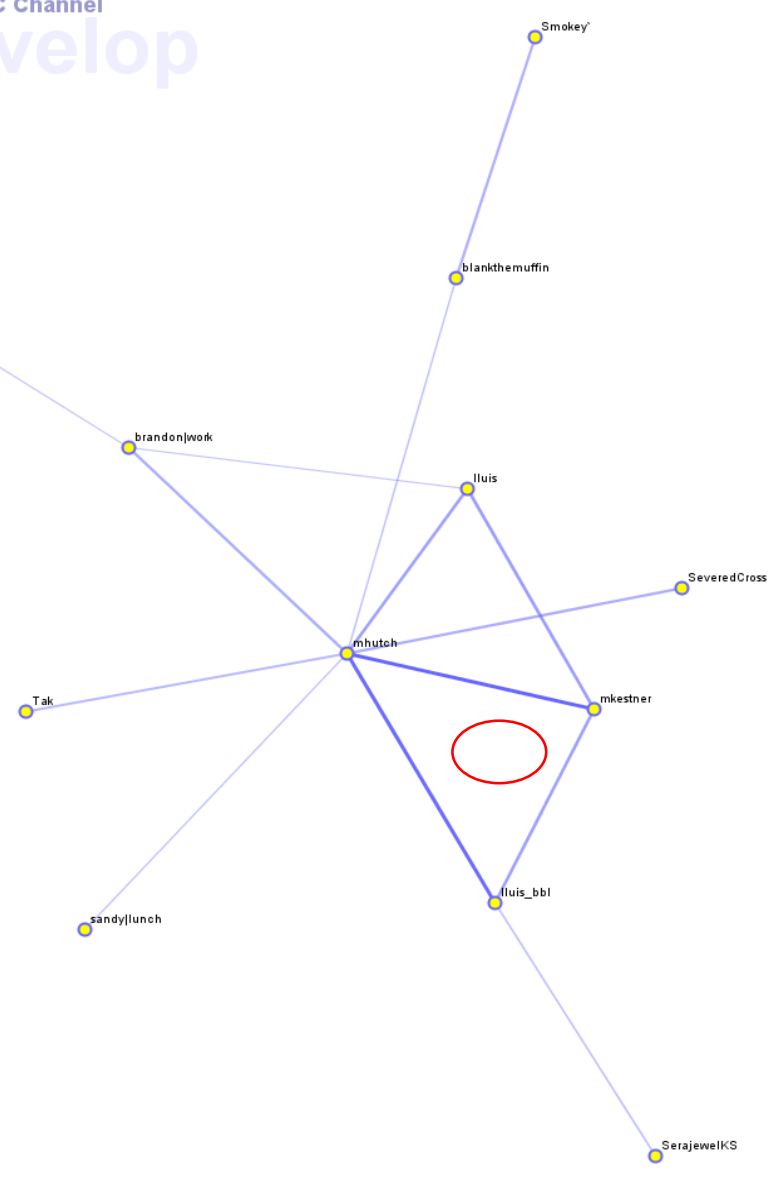


Figure 2

Formation of Sub-Groups with Temporal Shifts

A Social Network Diagram for an IRC Channel

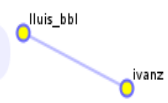

$0^{\text {directhex }}$
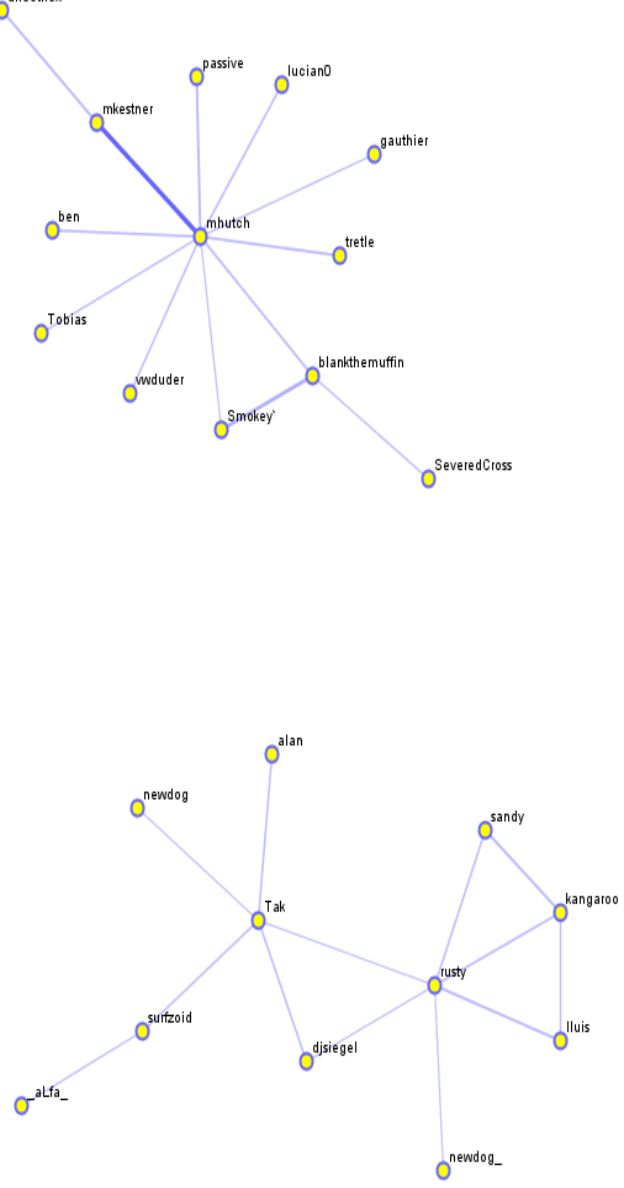
Figure 3

The IRC Conversation Map with the Log File and Social Network Graph Uploaded

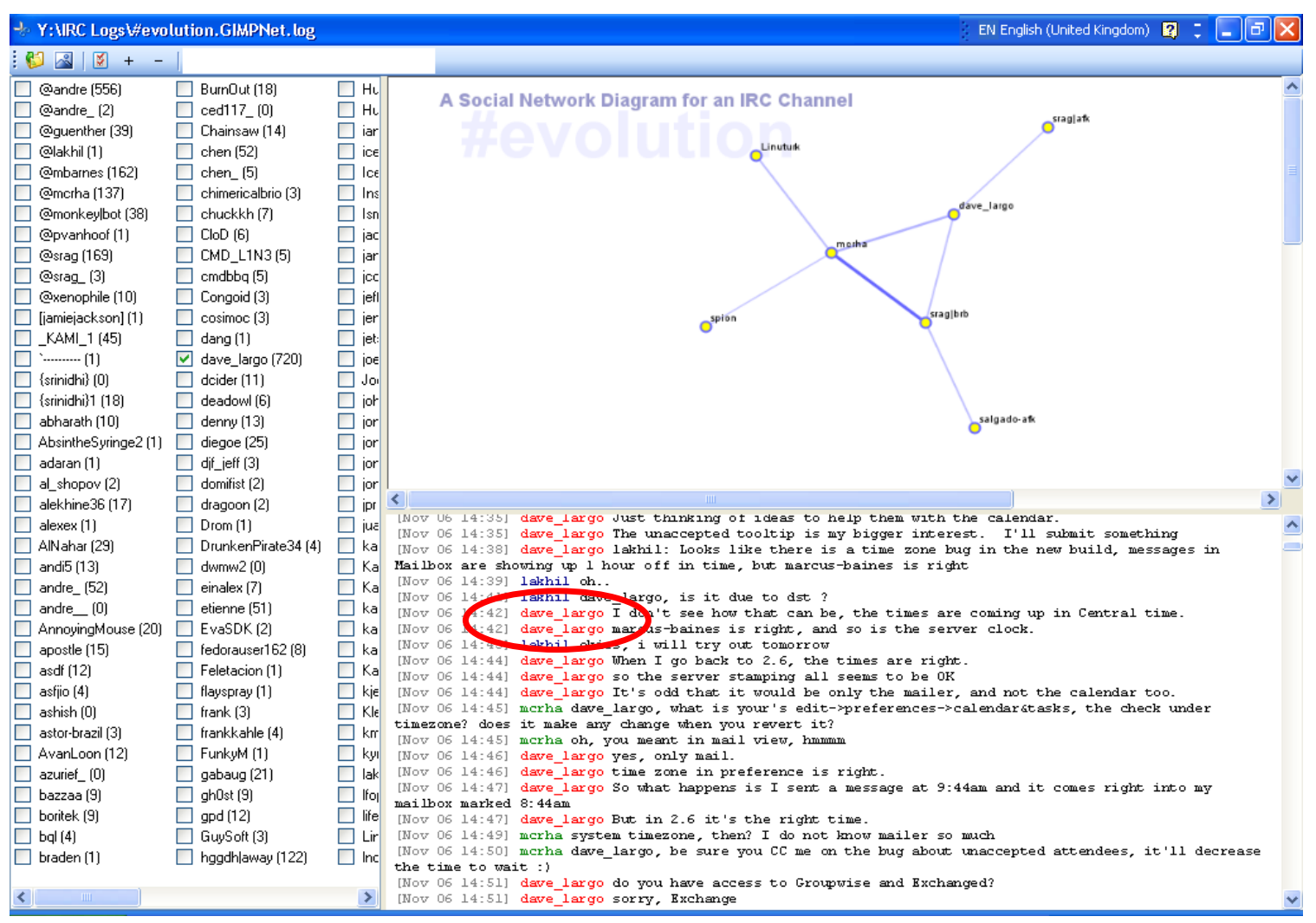

\section{Figure 4}

Keyword Search Analysis Made Possible

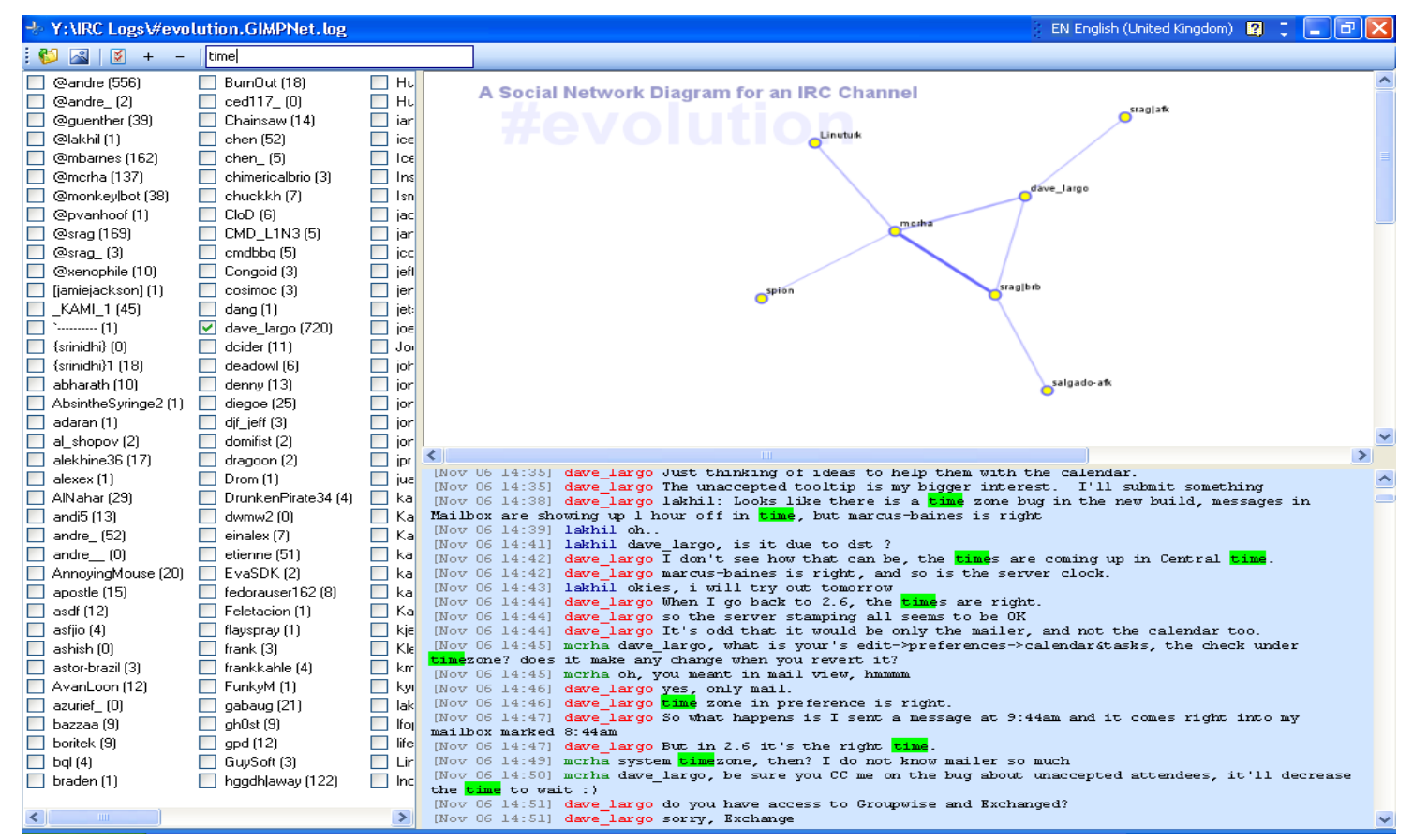


Figure 5

Modes of Analysis in the Data Collection Process

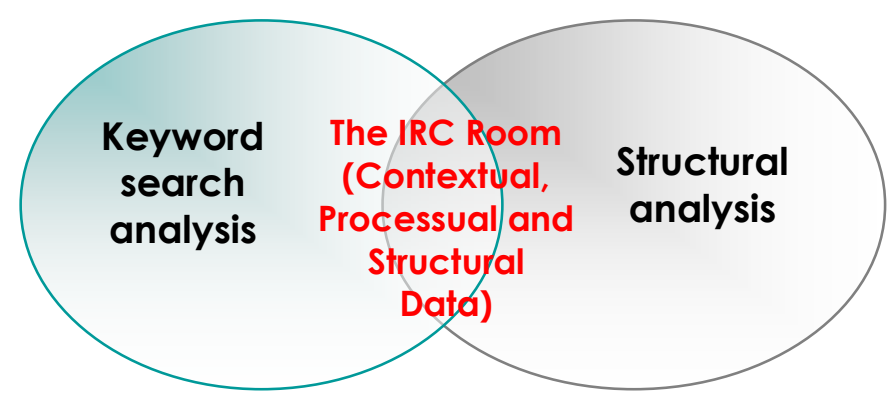

Figure 6

Illustration of Structural Analysis (Adapted from Ducheneaut, (2005)
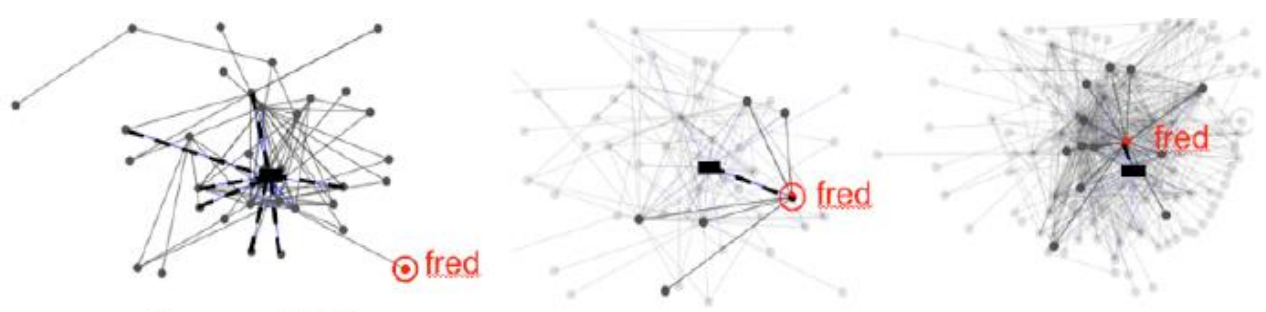

\section{Discussion}

The social science is currently passing through methodological changes infused by the Internet and the availability of vast amount of digital and social data that never existed before. Few research labs around the world are working on developing new research methods and tools that could support these new challenges. Bruno Latour; one of the prominent ANT gurus has recently joined the social science quest for new methods that could combine the qualitative with quantitative methods in order to collect and analyze the vast amount of data available.

This paper provides the experience of engaging in developing a digital research tool named the IRC Conversation Map. It is a generative and a graphical interface that facilitates the study of an online phenomenon and provides the opportunity to reflect critically on the data collected. It makes it possible to navigate through large scale conversations from social, spatial and semantic perspectives and to connect the macro and micro levels in a seamless analysis. In doing so, it provides empirical evidence to the digital debate in the social science and humanity.

\section{Conclusion}

The digital turn in social science and humanity field is emerging and taking shape with the scholarly efforts and contribution of sociology of science and technology, new media scholars, IM studies. This new field focus on exploiting the availability and 
affordances of different computing techniques to explore Internet-based and digital phenomena in new light (Bradley 2012). Engaging with the development, use and assessing the impact of virtual tools presents an opportunity for the IM field to significantly contribute and inform the social science and humanity.

It also brings this debate to invite researchers to engage in this phenomenon. As the experience presented in this paper shows, the digital quality-quantitative research requires multi-disciplinary perspective. Social science researchers need to work alongside computer scientists to develop the digital research instruments. IS researchers are more equipped to take this task with their accumulated knowledge in systems development, requirements gathering, virtual teams, and open source development. The IS field has also accumulated knowledge in interpretive research that examines the impact of information systems on working practices and output. This knowledge is important in this recent type of digital research as researchers need to be aware of the impact of their developed instrument on the collected and analysed data. Information systems field need to rise to inform the social science and humanity of the impact of using digital instruments. It could offer significant contribution in developing criteria for reporting and evaluating this type of digital research. The development of digital research evaluation criteria is a task IS researchers are capable of offering which would provide a significant advancement in that field. In conclusion, the rise of the digital research presents an opportunity for the IS field to directly and significantly contribute to the wider field of social science and humanity which this research is hoped to begin.

\section{References}

1. Bradley, J. (2012), No Job for Techies: Technical Contributions to Research in Digital Humanities, in Collaborative Research in the Digital Humanities, Farnham and Burlington: Ashgate.

2. Klang, M., Olsson, S. (1999), "Building communities online", In Proceedings of Fourth International Workshop on Cscw in Design, pp. 43-52.

3. Raymond, E. S. (1999), The Cathedral and the Bazaar, Cambridge, MA, O'Reilly.

4. Stallman, R. M. (2001), "Free Software: Freedom and Cooperation", (Transcript of speech), available at: www.gnu.org/events/rms-nyu-2001-transcript.html (02 March 2017)

5. Von Krogh, G., Spaeth, S., Lakhami, K. (2003), "Community, Joining, and Specialization in Open Source Software Innovation: A Case Study", Research Policy, Vol. 32 No. 7, pp. 1217-1241.

\section{About the author}

Meera Sarma, PhD, is a Senior Lecturer at Northumbria University, UK. She is studying the social construction of software development and innovation in virtual communities. Her research focuses on investigating hacking practices as it contributes to software innovation, by a social construction of the hacker community. Author can be contacted at meera.sarma@northumbria.ac.uk. 